\title{
Prevalence of Workplace Bullying and Its Associated Factors at a Multi-Regional Saudi Arabian Hospital: A Cross-Sectional Study
}

This article was published in the following Dove Press journal: Risk Management and Healthcare Policy

\author{
Khaled Al-Surimi (1D ${ }^{1,2}$ \\ Munirah Al Omar ${ }^{1,2}$ \\ Khalid Alahmary ${ }^{1,2}$ \\ Mahmoud Salam ${ }^{1-3}$ \\ 'Department of Health System \\ Management, College of Public Health \\ and Health Informatics, King Saud bin \\ Abdulaziz University for Health Sciences, \\ Riyadh, Saudi Arabia; ${ }^{2}$ King Abdullah \\ International Medical Research Center, \\ Riyadh, Saudi Arabia; ${ }^{3}$ Hariri School of \\ Nursing, American University of Beirut, \\ Beirut, Lebanon
}

Background: Workplace bullying (WPB) refers to any form of repeated and unreasonable verbal, physical or sexual harassment that an employee endures by a person or a group. In healthcare settings, practitioners are occasionally victims of WPB incidents. The aim of this study was to survey victims of WPB and determine factors associated with being a victim of WPB at a multiregional health care facility in Saudi Arabia.

Methods: This cross-sectional study was conducted in 2018, by distributing a selfadministered questionnaire via a private electronic mail to all fulltime healthcare practitioners within a multi-regional hospital in Saudi Arabia. Healthcare practitioners included physicians, nurses, allied healthcare professionals and pharmacists who reported being exposed to WPB in the past year. Study outcomes were the prevalence rate ratio of WPB and its associated factors, such as victim, perpetrator and incident characteristics.

Results: WPB has been reported by 684 participants. Perpetrators were mainly patients (36.1\%), their families/relatives $(29.5 \%)$, and hospital staff $(27.2 \%)$ or managers/supervisors (7.2\%). The type of WPB incident was mostly verbal abuse $(98.1 \%)$ followed by physical harassment (11.8\%) and sexual connotations (5.8\%). WPB was 30\% more prevalent among younger nurses and $24 \%$ less prevalent among higher educated nurses compared to their counter groups, $\mathrm{P}<0.001$ each. Among technicians and administrative employees, WPB was $54 \%$ more prevalent among females, 36\% more prevalent among the younger group, and $25 \%$ more prevalent among expatriate workers compared to their counter groups $\mathrm{P}<0.014$, $\mathrm{P}<0.001$ and $\mathrm{P}=0.017$, respectively. WPB was $20 \%$ less prevalent among higher educated allied health professionals, $\mathrm{P}=0.002$. Among physicians, WPB was $33 \%$ more prevalent among females, $\mathrm{P}=0.041$ and was $47 \%$ more prevalent among higher educated physicians compared to their counter groups, $\mathrm{P}=0.018$.

Conclusion: WPB might occur any time, anywhere and by any person within health care facilities. The prevalence of WPB varies within health occupational groups. Gender, age, educational level, and nationality were significantly associated factors with WPB.

Keywords: nurse, physician, pharmacist, bullying, verbal, physical, sexual

\section{Background}

Workplace Bullying (WPB) is generally known as a repeated and unreasonable mistreatment by a person or a group. ${ }^{1}$ WPB exemplifies itself in various forms such as verbal, physical or sexual harassment. ${ }^{2}$ The operational definition of bullying is a situation where one or several individuals, persistently and over a period of time are exposed to negative actions from one or several persons. ${ }^{3}$ Its prevalence varies across different industries all over the world, as some
Correspondence: Mahmoud Salam King Saud bin Abdulaziz University for Health Sciences, King Abdullah International Medical Research Center, Riyadh 22490, Saudi Arabia

$\mathrm{Tel}+96170054397$

Email mahmoudsalam@hotmail.com
Risk Management and Healthcare Policy 2020:13 1905-1914 
occupations, face a greater risk of exposure than others. ${ }^{4}$ Healthcare workers are occasionally victims of workplace hostility. For instance, in the United States, the rates of physical mistreatment against physicians and nurses were 16.2 per 1,000 and 21.9 per 1,000 employees, respectively. ${ }^{5}$ Furthermore, around $34.5 \%$ of emergency practitioners experience physical mistreatment, $71.6 \%$ of them encounter verbal abuse, and $44.4 \%$ report being bullied annually in one Iranian medical center. ${ }^{6}$ Apparently, WPB occurs because of a triad that constitutes a vulnerable victim, a bystander and a non-deterred perpetrator. $^{7}$

WPB among healthcare practitioners varies in its prevalence and characteristics across and within countries. ${ }^{8}$ In the European Union, $52 \%$ of the victimized healthcare employees have experienced bullying at work. ${ }^{9}$ Among US surgeons, one report showed that $40 \%$ of both residents and faculty reported being bullied, while 54.3\%-58.5\% witnessed a bullying incident. ${ }^{3}$ One study examining 3,700 respondents showed that $47 \%$ of physicians were bullied by other physicians, $29 \%$ by administrators, non-medical personnel, or patients, $17 \%$ by nurses, $4 \%$ by medical residents and fellows and $1 \%$ by medical students. ${ }^{10}$

Many experts view bullying as a direct reflection of misused power of the traditional hierarchy that exists in many healthcare organizations. ${ }^{9}$ For example, individuals with authority are often the perpetrators who bully their subordinates, solely driven by the virtue of their position. ${ }^{11}$ In addition, the absence of stringent laws and policies sometimes make employers pay less attention to prevent it. ${ }^{12}$ In a systematic review paper, WPB was a predictor of various mental health problems and sleep disturbances, ${ }^{13}$ yet sadly, about $29 \%$ of victims remain silent about their WPB experience. ${ }^{14}$ However, the most serious form of socioeconomic consequences of WPB is when practitioners who try to stop it voluntarily decide to leave their job. ${ }^{15}$ Unfortunately, WPB in healthcare settings affects teamwork, jeopardizes its integrity, ${ }^{11}$ and negatively affects patients' safety and quality of care. ${ }^{16}$ Intimidating and disruptive behaviors associated with WPB fuel medical errors and lead to adverse outcomes. ${ }^{16,17}$ In addition to being a dramatic threat to patient safety, ${ }^{18}$ WPB weakens staff morale and increases absenteeism, leading to higher turnover rates of qualified staff. $^{19}$

Although several studies have extensively examined the prevalence and characteristics of WPB, it is unfortunate that this phenomenon has not been addressed sufficiently in some Middle Eastern settings. For instance, in Saudi Arabia, studies on WPB are few, which leaves numerous gaps in the body of knowledge. ${ }^{16}$ The Saudi Arabian community is distinctive compared to Western settings, in terms of race and ethnicity, with deeply rooted traditions framed by tribal bounds and religious constraints. ${ }^{20}$ The Saudi Arabian health industry is in high demand for international expatriates, and hospitals in this region employ healthcare practitioners from all over the world. ${ }^{21}$ In addition to the rules and regulations that ban WPB, the culture and religion supposedly create further restraints on harming other individuals. On the other hand, victims of WPB in such settings might under report such unfortunate events, driven by the fear of being laid off. ${ }^{22}$ Therefore, it is imperative for different healthcare settings to detect any case of WPB, to analyze its characteristics and to determine high-risk work environments. The aim of this study was to survey victims of WPB and determine factors associated with being a victim of WPB at a multiregional health care facility in Saudi Arabia.

\section{Methods}

\section{Study Design and Setting}

This was a cross-sectional correlation study based on an anonymous self-reported survey. It is the second part of a project and a follow-up paper to a previous publication that determined to what extent healthcare practitioners worry about WPB and whether it affects the quality of care and patient safety from their perception. ${ }^{16}$ The targeted setting comprised four hospitals situated in various geographical regions in Saudi Arabia (Two Eastern, one Central and one Western) all affiliated with one governmental institution. It employs more than 7,000 employees with a total bed capacity exceeding 1,000 beds. It has been accredited by the Joint Commission International several times. The health care industry in Saudi Arabia employs local Saudi healthcare workers who serve a relatively religious, tribal, and conservative culturally oriented community. However, due to the severe shortage in the work force, an influx of expatriates of various races, ethnicities and cultures from all over the world has been observed. ${ }^{21}$

\section{Study Participants}

The accessible population was fulltime healthcare practitioners (physicians, nurses, pharmacists, administrative employees and technicians) of various career levels and registered with the Saudi Commission for Health Care 
Specialties. These participants were asked if they were victims of WPB by reporting their experience with any form of violence regardless of its magnitude and duration in the past year. They were asked to report the details of their WPB experience (type of violence, perpetrator, place, initial reaction, method of reporting). Perpetrators were either healthcare practitioners, patients or visitors of patients who committed various forms of violent acts as perceived and reported by the victims. Due to the sensitivity of this topic and to maximize on the participants' privacy, the data collection package consisted of a letter of invitation, an informed consent and an English language survey, all electronically accessible through a hyperlink sent through a private electronic mail. The invitation letter clarified the objectives of the study to the invitees and informed them that their participation will contribute to the body of science on WPB. A written informed consent was secured by electronically ticking on an "agreement to participate" statement. By convenience, all participants were exposed to the study, as the survey was distributed via a mass email service in 2018 , twice with a one-month interval. Those who participated the first time were instructed to refrain from participating again. A disabled tracking of the filed surveys to the participants' email addresses insured confidentiality of participants.

\section{Data Collection Tool}

The survey measured both participants' and WPB's characteristics. Participants' characteristics were commonly assessed variables in the literature associated with WPB. These included gender (male vs female), age (years), marital status (single, married, separated/widowed), level of education (diploma, bachelor, masters or $\mathrm{PhD}$ degree), nationality (Saudis vs, Expatriates), work duration (years) and occupation (nurses, technicians, administrative, physician, pharmacists). While there is no commonly reported cut-off for age and work duration when investigating WPB, we have categorized them based on ten-year intervals, before collapsing them into binary groups. Participants with a diploma or bachelor's degree were grouped under lower education, while those with Master's or $\mathrm{PhD}$ degree were grouped under higher education. Technicians and administrative practitioners were grouped under the allied health category of occupation.

As per the World Health Organization (WHO), WPB was defined as a multifaceted form of mistreatment, characterized by a repeated exposure to physical and/or emotional aggression. Physical bullying includes an assault (with or without injury) to the individual's body or property, such as beating, kicking, spitting, pinching, pushing, or using a rude body language. Verbal bullying involves the use of offensive words through teasing, name-humiliating, and unacceptable sexual comments. ${ }^{23}$ Participants who met the criteria of $\mathrm{WPB}^{23}$ were counted as victims. WPB characteristics were mainly the identity of the perpetrator (patient, family of patient, employees), the timing, and the place of the incidents as reported by the victims. In addition, victims were questioned about their personal reactions or responses to WPB, if they received any support, as well as the underlying cause of WPB as perceived by the participant. The consequences of WPB against the perpetrator were also recorded. The feasibility and content validity of the questionnaire were tested before being piloted among 16 practitioners. Their subjective feedback was analyzed, and modifications were made to enhance the comprehension of the survey.

\section{Statistical Analysis}

Data entry and analyses were conducted using SPSS v.26 (IBM, NY). Participants' and WPB incident characteristics were presented in frequencies (n) and percentages (\%). Factors associated with being a victim were tested using Pearson's chi-square. Since the prevalence of the outcome was high, the prevalence rate ratio (PRR) was presented as the measure of association between exposures and outcomes. ${ }^{24}$ PRR is calculated by dividing the prevalence rate of WPB within one subgroup over the prevalence rate of WPB in the counter group. Higher PRR indicated how large is the prevalence of WPB in one group (percentage ratio) relative to the other group of the same characteristics. PRRs and their $95 \%$ confidence interval $(95 \% \mathrm{CI})$ were obtained by conducting Poisson regression analyses (robust error estimation) to model the relationship between the study exposures (gender, age, marital status, nationality, education) and WPB as a binary outcome. Regression analyses were stratified by occupation (nurses, allied health, physicians) due to the differences in their scope of practice, chain of command, level of teamwork, and type of workload for each occupation. Statistical significance was set at $\mathrm{P}$-value $<0.05$.

\section{Ethics Approval and Consent to Participate}

A self-explanatory letter of invitation to participate in this study was presented to each of the participants. All participants gave written informed consents for their enrollment in the study presented in this manuscript with full knowledge of the possible risks and benefits of participation. Participants 
consented by ticking "agree", indicating their agreement to provide their feedback for this research study. Study was approved by the Institutional Review Board at the Saudi Ministry of National Guard - Heath Affairs (Protocol \# SP18/057/R). This study followed the recommendations of the International Conference on Harmonization for Good Clinical Practice (ICH-GCP) and in compliance with the Declaration of Helsinki.

\section{Results}

\section{Participant and WPB Characteristics}

A total of 1,074 participants responded to the survey, among whom $684(63.7 \%)$ admitted to being victims of WPB in the past year and agreed to disclose its details.
The majority of participants were females $(85.8 \%)$, while $67.6 \%$ were $\geq 30$ years of age. Almost half of the nurses admitted to being victims (48.3\%), administrative staff $(22.7 \%)$, physicians $(13.6 \%)$, technicians $(12.9 \%)$ and pharmacists $(2.5 \%)$. Other participants' characteristics are presented in Table 1.

WPB perpetrators were mainly patients $(36.1 \%)$, their relatives or families $(29.5 \%)$, work colleagues $(27.2 \%)$ or managers/supervisors $(7.2 \%)$. The type of incident was mainly verbal abuse (98.1\%), physical harassment (11.8\%) and sexual connotation (5.8\%). WPB mainly occurred at day shifts $(80.3 \%)$ and at working stations/ offices $(52.6 \%)$. Almost half of the victims decided to inform a friend or a family member $(48.4 \%)$ and/or report to a senior staff $(28.8 \%)$. Most of the nurses claimed that

Table I Association of Workplace Bullying With Sample Characteristics

\begin{tabular}{|c|c|c|c|c|}
\hline & \multicolumn{3}{|c|}{ Exposure to Workplace Bullying } & \multirow[t]{2}{*}{ P-value } \\
\hline & $\begin{array}{l}\text { Yes } \\
\text { n (\%) } \\
684(63.7)\end{array}$ & $\begin{array}{l}\text { No } \\
\text { n (\%) } \\
390(36.3)\end{array}$ & $\begin{array}{l}\text { Total } \\
\text { n (\%) } \\
1074(100)\end{array}$ & \\
\hline \multicolumn{5}{|l|}{ Gender } \\
\hline Male & $74(48.7)$ & $78(5 \mid .3)$ & $152(14.2)$ & $<0.001 *$ \\
\hline Female & $610(66.2)$ & $312(33.8)$ & $922(85.8)$ & \\
\hline \multicolumn{5}{|l|}{ Age (years) } \\
\hline$<30$ & $269(77.3)$ & $79(22.7)$ & $348(32.4)$ & $<0.001 *$ \\
\hline $30-40$ & $139(46.8)$ & $158(53.2)$ & $297(27.7)$ & \\
\hline$>40$ & $276(64.3)$ & $153(35.7)$ & $429(39.9)$ & \\
\hline \multicolumn{5}{|l|}{ Marital status } \\
\hline Single/separated & $373(69.9)$ & $161(30.1)$ & $536(49.7)$ & $<0.001 *$ \\
\hline Married & $311(57.6)$ & $229(42.4)$ & $540(50.3)$ & \\
\hline \multicolumn{5}{|l|}{ Educational status } \\
\hline Diploma's/Bachelor's degree & $549(63.3)$ & $318(36.7)$ & $867(80.7)$ & 0.610 \\
\hline Master's/PhD degree & $135(65.2)$ & $72(34.8)$ & $207(19.3)$ & \\
\hline \multicolumn{5}{|l|}{ Nationality } \\
\hline Local & $165(56.5)$ & $127(43.5)$ & $292(27.2)$ & $0.003^{*}$ \\
\hline Expatriate & $519(66.4)$ & $263(33.6)$ & $782(72.8)$ & \\
\hline \multicolumn{5}{|l|}{ Work experience (years) } \\
\hline$\leq 10$ & $4 I I(70.0)$ & 176(30.0) & $587(54.7)$ & $<0.001 *$ \\
\hline$>10$ & $273(56.1)$ & $2 \mid 4(43.9)$ & $487(45.3)$ & \\
\hline \multicolumn{5}{|l|}{ Occupation } \\
\hline Nurses & $336(64.7)$ & $183(35.3)$ & $519(48.3)$ & $<0.001 *$ \\
\hline Technicians & $74(53.2)$ & $65(46.8)$ & $139(12.9)$ & \\
\hline Administrative & $166(68.0)$ & $78(32.0)$ & $244(22.7)$ & \\
\hline Physician & $100(68.5)$ & $46(31.5)$ & $146(13.6)$ & \\
\hline Pharmacists & $8(30.8)$ & $18(69.2)$ & $26(2.5)$ & \\
\hline
\end{tabular}

Note: *Signifies statistical significance using Pearson's chi-square test.

Abbreviations: $n$, frequency; \%, percentage. 
the cause of the incident was misunderstanding (77\%), yet $58.2 \%$ claimed that it was due to a lack of disciplinary actions that holds perpetrators accountable to their behavior. As reported by the WPB victims, only $33.6 \%$ of perpetrators received verbal warning, while no action was taken in $23.4 \%$ of the incidents. Other WPB characteristics have been enlisted in Table 2 .

\section{Workplace Bullying Across Sample Characteristics}

WPB was significantly more prevalent among female healthcare practitioners $66.2 \%$, compared to male practitioners $(48.7 \%), \mathrm{P}<0.001$. Healthcare practitioners $<30$ years were also more likely to be bullied $(77.3 \%)$ compared to older practitioners $\geq 30$ years of age $(57.2 \%)$, $\mathrm{P}<0.001$. Single or separated healthcare practitioners were also more likely to be exposed to WPB (69.9\%), compared to married ones (57.6\%), $\mathrm{P}<0.001$. WPB was more prevalent among expatriate non-Saudi healthcare practitioners $(66.4 \%)$, and practitioners with $\leq 10$ years work experience $(70.0 \%), \mathrm{P}=0.003$ and $\mathrm{P}<0.001$, respectively. In terms of occupation, $64.7 \%$ of nurses, $68.5 \%$ of physicians, $68.0 \%$ of administrative employees, $53.2 \%$ of technicians and $30.8 \%$ of pharmacists were exposed to WPB, Table 1. The percentage distribution of perpetrator with the occupation of WPB victims is illustrated in Figure 1.

\section{Factors Associated with Being a Victim of WPB}

Poisson binary regression analyses were conducted stratified by occupation. Pharmacists were not included in the regression model analysis due to the low sample size $(\mathrm{n}=26)$. WPB was 30\% (95\% CI: $14 \%-48 \%$ ) more prevalent among younger nurses and 24\% (95\% CI: $21 \%-34 \%$ ) less prevalent among higher educated nurses compared to their counter groups, $\mathrm{P}<0.001$ each. Among technicians and administrative employees, WPB was 54\% (95\% CI: 9\%-117\%) more prevalent among females, 36\% (95\% CI: 16\%-61\%) more prevalent among the younger group, and $25 \%$ (95\% CI: $4 \%$ $50 \%)$ more prevalent among expatriates compared to their counter groups, $\mathrm{P}<0.014, \mathrm{P}<0.001$ and $\mathrm{P}=0.017$, respectively. WPB was $20 \%$ (95\% CI: 9\%-117\%) less prevalent among higher educated allied health, $\mathrm{P}=0.002$. Among physicians, WPB was 33\% (95\% CI: $1 \%-76 \%)$ more prevalent among females compared to male physicians, $\mathrm{P}=0.041$ and it was $47 \%$ (95\% CI:7\%-104\%) more prevalent among higher
Table 2 Distribution of Workplace Bullying Incident Characteristics

\begin{tabular}{|c|c|}
\hline & $\begin{array}{l}n(\%) \\
684(100.0)\end{array}$ \\
\hline \multicolumn{2}{|l|}{ Type of bullying* } \\
\hline Verbal & $67 \mid(98.1)$ \\
\hline Physical & $81(11.8)$ \\
\hline Sexual & $40(5.8)$ \\
\hline \multicolumn{2}{|l|}{ Perpetrator of incidents } \\
\hline Patient & $247(36.1)$ \\
\hline Relatives of patients (visitors) & $202(29.5)$ \\
\hline Manager or supervisor & $49(7.2)$ \\
\hline Employees & $186(27.2)$ \\
\hline \multicolumn{2}{|l|}{ Time of incident } \\
\hline Day shift & $549(80.3)$ \\
\hline Evening shift & $46(6.7)$ \\
\hline Night shift & $89(13.0)$ \\
\hline \multicolumn{2}{|l|}{ Area of incident } \\
\hline Patient room & $142(20.9)$ \\
\hline Treatment room/Clinic & 157(23.0) \\
\hline Working station/office & $360(52.6)$ \\
\hline Nonclinical areas & $24(3.5)$ \\
\hline \multicolumn{2}{|l|}{ Reaction to workplace bullying* } \\
\hline Told friends/family & $33 \mathrm{I}(48.4)$ \\
\hline Reported to senior staff member & 197(28.8) \\
\hline Told the person to stop & $183(26.8)$ \\
\hline Told a colleague & $180(26.3)$ \\
\hline Tried to defend self physically & $124(18.1)$ \\
\hline Completed incident or accident form & $76(11.1)$ \\
\hline Sought counseling & $55(8.0)$ \\
\hline Transferred to another position & $46(6.7)$ \\
\hline No action, pretended it never happened & $71(6.6)$ \\
\hline \multicolumn{2}{|l|}{ Causes of workplace bullying* } \\
\hline Misunderstanding & $527(77.0)$ \\
\hline Lack of discipline to hold people accountable about their behavior & $398(58.2)$ \\
\hline Concern of patients & $279(40.8)$ \\
\hline Communication or Language barriers & $267(39.0)$ \\
\hline Lack of explicit rights or procedure & $118(17.3)$ \\
\hline lllness & $106(15.5)$ \\
\hline Personal problem of coworker & $96(14.0)$ \\
\hline Scapegoat for medical dispute & $26(3.8)$ \\
\hline Poor training on WPB & $24(3.5)$ \\
\hline Fault of oneself & $23(3.4)$ \\
\hline Drinking problem & $15(2.2)$ \\
\hline \multicolumn{2}{|l|}{ Consequences to the perpetrator } \\
\hline None & 160(23.4) \\
\hline Verbal warning issued & $230(33.6)$ \\
\hline Written warning & $83(12.1)$ \\
\hline Care discontinued & $58(8.5)$ \\
\hline Counseling & $10(1.5)$ \\
\hline Reported to police (prosecution) & $9(1.3)$ \\
\hline Do not know & $134(19.6)$ \\
\hline
\end{tabular}

Note: *Non-mutually exclusive.

Abbreviations: $\mathrm{n}$, frequency; \%, percentage. 


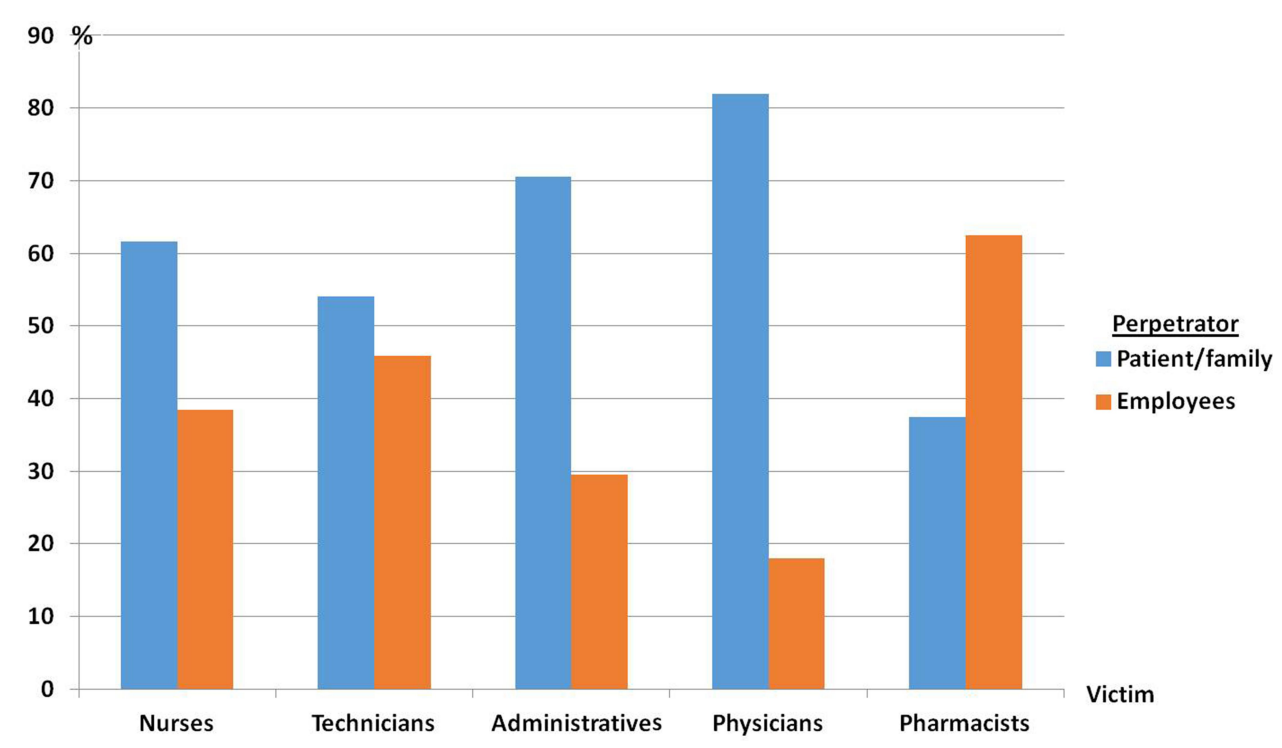

Figure I Perpetrator of Work Place Bullying with the occupation of victims.

educated physicians compared to their counter group, compared to males. This finding was similar to the report $\mathrm{P}=0.018$ (Table 3).

\section{Discussion}

Hospital administrators should be cautioned that WPB of a Swedish health care system where victims of WPB were predominantly women $(90 \%){ }^{8}$ Gender plays an important role in predicting $\mathrm{WPB} ;{ }^{25}$ however, gender is more prevalent among female healthcare practitioners should not be accounted as a single precursor to WPB at health care facilities. In this setting, occupation was

Table 3 Poisson Binary Regression Analyses of Factors Associated with Being a Victim of Workplace Bullying Stratified by Victims' Occupation

\begin{tabular}{|c|c|c|c|c|c|c|}
\hline & \multicolumn{2}{|l|}{ Nurse } & \multicolumn{2}{|c|}{$\begin{array}{l}\text { Allied Health } \\
\text { (Technicians and Administrative) }\end{array}$} & \multicolumn{2}{|l|}{ Physicians } \\
\hline & PRR $[95 \% \mathrm{Cl}]$ & P-value & PRR $[95 \% \mathrm{Cl}]$ & P-value & PRR $[95 \% \mathrm{Cl}]$ & P-value \\
\hline $\begin{array}{l}\text { Gender } \\
\text { Male }^{(\text {Ref }} \\
\text { Female }\end{array}$ & $0.83[0.67-1.03]$ & 0.083 & $1.54[1.09-2.17]$ & $0.014^{*}$ & $1.33[1.01-1.76]$ & $0.04 I^{*}$ \\
\hline $\begin{array}{l}\text { Age } \\
\qquad \begin{array}{l}\geq 30^{(\text {Ref })} \\
\quad<30\end{array}\end{array}$ & $1.30[1.14-1.48]$ & $<0.00 I^{*}$ & $1.36[1.16-1.61]$ & $<0.00 I^{*}$ & I.I2[0.85-I.46] & 0.428 \\
\hline $\begin{array}{l}\text { Marital status } \\
\text { Married (Ref) } \\
\text { Single }\end{array}$ & I.I0[0.95-1.27] & 0.206 & $0.95[0.80-1.13]$ & 0.587 & $1.21[0.91-1.60]$ & 0.187 \\
\hline $\begin{array}{c}\text { Nationality } \\
\text { local (Ref) } \\
\text { Expatriate }\end{array}$ & I.14[0.94-I.38] & 0.195 & $1.25[1.04-1.50]$ & $0.017^{*}$ & $1.20[0.98-1.48]$ & 0.084 \\
\hline $\begin{array}{l}\text { Education } \\
\text { Lower (Ref) }^{\text {Higher }}\end{array}$ & $0.76[0.66-0.88]$ & $<0.00 I^{*}$ & $0.80[0.69-0.92]$ & $0.002^{*}$ & $1.47[1.07-2.04]$ & $0.018^{*}$ \\
\hline Intercept & $0.76[0.57-1.03]$ & 0.076 & $0.4 \mathrm{I}[0.29-0.58]$ & $<0.00 I^{*}$ & $0.31[0.22-0.44]$ & $<0.001 *$ \\
\hline
\end{tabular}

Note: *Significance of $\mathrm{P}<0.05$.

Abbreviations: ${ }^{(\mathrm{Ref})}$, reference group; \%, percentage; $\mathrm{Cl}$, confidence interval; PRR, prevalence rate ratio. 
associated with WPB. This phenomenon can be attributed to the scope of practice. Some healthcare practitioners, such as nurses or allied health employees are more involved in bedside care and differ in their chain of command compared to physicians or pharmacists. ${ }^{26}$ Nurses and allied healthcare practitioners report to unit managers, nurse coordinators, shift supervisors, and other health disciplines. They operate in larger teams and their workload is more physically demanding, even though they are limited to fewer patients when compared to physicians or pharmacists. ${ }^{27}$ Authors believe that this difference in scope of practice and work relationships contributed to the variation in WPB incidents between them. On the other hand, physicians/pharmacists tend to rotate between various hospital wards. They usually communicate more with patients/families, especially when it has to do with reporting medical diagnoses, clinical progress and critical lifesaving decisions. Therefore, WPB among physicians/ pharmacists might have been due to disrupted communication with the patients/families, lack of patients' expectations, or patients' misinterpretation of the delivered messages. In this setting, bullied physicians/pharmacists claimed that it was due to miscommunication with patients $(27.7 \%)$ or misinterpretation of medical instructions (86\%). Any disrupted communication between families and health care teams eventually results in stress, temper, lack of confidence, and, subsequently, violence. ${ }^{28}$

Age was a significant factor associated with WPB in this setting, as WPB was more prevalent (77.3\%) among younger healthcare practitioners $(<30$ years). WPB was also more prevalent $(70 \%)$ among less experienced employees $(<10$ years). Age and work experience are collinear variables. We believe that fresh graduates and junior practitioners have not sufficiently developed their interpersonal work relationships or communication skills, which explains why $70 \%$ of WPB was observed among this group. A synthesis of evidence from 16 studies showed that newly graduated employees were at higher risk of being exposed to a negative workplace behavior. ${ }^{29}$ In this setting, the young and less experienced practitioners who reported WPB were probably incapable of delivering the message properly to patients or their families (33.4\%). Furthermore, in one study, nurses who have been abused by a patient or family member failed to find support from their managers. ${ }^{30}$ This was comparable with findings in this setting, as $30.6 \%$ of nurses who were bullied reported a lack of support from management. In addition, authors found that junior practitioners in this setting were subject to criticizing comments from senior practitioners $(11.9 \%)$. The stressful work environment might be challenging to them, which makes them lose focus on properly handling a WPB incident when the need arises. For instance, it has been reported that practitioners usually focus on their own patient assignments with little time or interest in conflict resolution. ${ }^{30}$ Hectic duties and stressful work environments witnessed by these practitioners might trigger a loss of temper. In this setting, $39 \%$ of practitioners reported that the lack of communication contributed to WPB, while $14 \%$ stated that it was due to poor collaboration. Furthermore, healthcare practitioners regularly find short-notice and multiple batch admissions or discharges as major stressors, especially if it is not well coordinated with the admission office. Unmet patient/family expectations, such as lack of vacant beds or absence of resources, will eventually create a chaotic work environment where WPB might arise. ${ }^{30}$ In this setting, $40.8 \%$ of practitioners who were bullied claimed that it was due to their inability to satisfy patient/family needs.

WPB was also more prevalent among expatriate healthcare practitioners compared to local Saudi employees. Victims of WPB in one setting reported that these incidents arose due to cultural difference or language barriers. ${ }^{31}$ In this setting, English language is the predominant language used among healthcare practitioners, yet Arabic language is the official language for the public community. Non-Arabic speaking expatriate healthcare practitioners strive to communicate with their patients using simplified, unstructured Arabic dialect. This factor might have aggravated a potential misunderstanding that escalated to WPB, simply due to a failure in expressing the true thoughts, as reported by $77 \%$ of bullied victims in this study. Currently, there have been no previously published studies that related WPB to language and cultural differences between healthcare practitioners and patients/their relatives. Accordingly, hospital administrators are encouraged to disseminate educational offerings throughout the health care settings about proper work relationships and professional communication.

\section{Limitations}

A number of limitations have been observed in this study. Authors believe that WPB is an under-reported phenomenon, so the recruited sample might not be exhaustive in its representativness. We suspect that some participants might have been exposed to a certain form of harassment once, and perceived it is as bullying. Nevertheless, some 
victims might have been harassed once, yet from different patients, visitors or employees. This makes the perpetrator not necessarily restricted to one confined incident. In addition, there is a possibility that some participants had experience with WPB or were more likely to be interested to respond to the survey compared to non-participants. Moreover, although participants were not involved in the recruitment of other study participants, there is a chance that some might have shared the link of the survey with other participants who were not originally invited to participate. Disclosing the WPB incidents to hospital administrators necessitates a supportive work culture and a transparent reporting system that insures the privacy and safety of WPB victims. Another limitation is recall bias, taking into account the retrospective design of the study. However, authors believe that the details of WPB, whether it is an isolated or a repetitive incident, are unlikely to be forgotten by the victims. In addition, certain conflicts, among employees or between employees and patients/their families, might have been misinterpreted by the victims as an act of aggression or bullying. Furthermore, life factors such as social/financial stressors, previous mental/psychological disorders or even prior traumas might have elicited a WPB incident from the perpetrator's side or increased the vulnerability from the victim's side. Therefore, in-depth analyses of the true causes and implications of WPB incidents require a more personal qualitative research methodological approach, which was beyond the scope of this study.

\section{Conclusion}

WPB might occur any time, anywhere and by any person within healthcare facilities. The prevalence of WPB varies within occupational groups. Gender, age, educational level, and nationality were all factors associated with WPB. The leading form of WPB in this setting was verbal abuse that mainly occurred during day shifts. WPB was more prevalent among female healthcare practitioners, $<30$ years of age, and expatriates. Among nurses, it was more prevalent among those $<30$ years old, and those with advanced educational levels. Among allied healthcare professionals (technicians and administrative staff), female gender, young age, expatriate, and advanced education were factors associated with WPB incidents. WPB was more prevalent among female physicians and those with higher educational degrees. WPB incidents were more likely to occur at work stations, followed by treatment rooms or clinics.
WPB is unfortunate by all means. It is the employees' and patients' right, as well as the duty of all hospital administrators, to promote a safe working environment, free of any harassment type. This study analyzed actual WPB incidents in attempt to identify occupational groups with high prevalence rates of WPB. Accordingly, action plans can be launched to educate and train vulnerable groups, and to closely monitor work environments. Identifying high-risk work environments includes detecting, reporting and resolving early warning signs of WPB. Regrettably, some believe that interventions to prevent WPB are ineffective, with emphasis on the fact that WPB is being primarily facilitated by organizational causes and culture that hinders the formation of collegiality and trust among employees. ${ }^{32}$

\section{Article Summary}

\section{Strengths and Limitations}

- Workplace bullying has not been addressed sufficiently in Middle Eastern settings.

- It is imperative to all healthcare settings to detect any case of WPB, to analyze its characteristics, determine high-risk groups, and take the right action.

- This study analyzed the various characteristics of WPB incidents at a multi-regional healthcare facility in Saudi Arabia.

- Most WPB incidents remain under reported, so the recruited sample might not be exhaustive .

- Certain conflicts, among employees or between employees and patients/their families, might have been misinterpreted by the victims as an act of aggression or bullying.

\section{Key Points}

- Leading type of WPB in this healthcare setting is verbal abuse that occurred during day shifts.

- WPB seems more prevalent among females, young age group, and expatriates.

- WPB is more likely to occur at work stations or offices.

- WPB perpetrators were mainly patients, relatives of patients, employees or managers/supervisors.

\section{Abbreviations}

95\% CI, 95\% confidence interval; PRR, prevalence rate ratio; WPB, workplace bullying; WHO, World Health Organization. 


\section{Data Sharing Statement}

The datasets generated and/or analyzed during the current study are not publicly available due to the institutional rules and regulations but are available from the corresponding author on reasonable request.

\section{Acknowledgments}

This study was approved and monitored by King Abdullah International Medical Research Center, King Saud bin Abdulaziz University for Health Sciences, Riyadh, Saudi Arabia. The authors would like to thank Mr. Mohamad AlAssiri and Mrs. Lara Afesh for their tremendous support. The authors are grateful to the participants who shared the details of unfortunate WPB incidents. We hereby presented this valuable contribution for the sake of protecting healthcare practitioners from WPB incidents in the future.

\section{Author Contributions}

All authors made substantial contributions to conception and design, acquisition of data, or analysis and interpretation of data; took part in drafting the article or revising it critically for important intellectual content; agreed to submit to the current journal; gave final approval of the version to be published; and agree to be accountable for all aspects of the work.

\section{Funding}

There is no funding to report.

\section{Disclosure}

The authors declare that they have no competing interests for this work.

\section{References}

1. Al-Ghabeesh SH, Qattom H. Workplace bullying and its preventive measures and productivity among emergency department nurses. BMC Health Serv Res. 2019;19(1):445. doi:10.1186/s12913-0194268-x

2. Hollis LP. Bully university? The cost of workplace bullying and employee disengagement in American higher education. Sage Open. 2015;5(2):2158244015589997.

3. Pei KY, Hafler J, Alseidi A, Slade MD, Klingensmith M, Cochran A. National assessment of workplace bullying among academic surgeons in the US. JAMA Surg. 2020;155(6):524. doi:10.1001/jamasurg.2020. 0263

4. Feijó FR, Gräf DD, Pearce N, Fassa AG. Risk factors for workplace bullying: a systematic review. Int $J$ Environ Res Public Health. 2019;16(11):1945. doi:10.3390/ijerph16111945

5. O'Rourke M, Wrigley C, Hammond S. Violence within mental health services: how to enhance risk management. Risk Manag Healtho Policy. 2018;11:159. doi:10.2147/RMHP.S131834
6. Saleh LA, Niroumand S, Dehghani Z, Saleh TA, Mousavi SM, Zakeri H. Relationship between workplace violence and work stress in the emergency department. J Inj Violence Res. 2020;12(2).

7. Cantone E, Piras AP, Vellante M, et al. Interventions on bullying and cyberbullying in schools: a systematic review. Clin Pract Epidemiol Ment Health. 2015;11:58. doi:10.2174/1745017901511010058

8. Rahm G, Rystedt I, Wilde-Larsson B, Nordström G, Strandmark KM. Workplace bullying among healthcare professionals in Sweden: a descriptive study. Scand J Caring Sci. 2019;33(3):582-591. doi:10.1111/scs.12633

9. Ariza-Montes A, Muniz N, Montero-Simó M, Araque-Padilla R. Workplace bullying among healthcare workers. Int J Environ Res Public Health. 2013;10(8):3121-3139. doi:10.3390/ijerph10083121

10. Medscape. Medscape report finds physicians are sexually harassed on the job. June, 2018.

11. Bjørkelo B, Einarsen S, Matthiesen SB. Predicting proactive behaviour at work: exploring the role of personality as an antecedent of whistleblowing behaviour. $J$ Occup Organ Psychol. 2010;83 (2):371-394. doi:10.1348/096317910X486385

12. Rivara F, Le Menestrel S. Preventing Bullying Through Science, Policy, and Practice. The National Academies Press; 2016.

13. Nielsen MB, Harris A, Pallesen S, Einarsen SV. Workplace bullying and sleep-a systematic review and meta-analysis of the research literature. Sleep Med Rev. 2020;101289.

14. Namie G, Namie R. US workplace bullying: some basic considerations and consultation interventions. Consult Psychol J: Pract Res. 2009;61(3):202. doi:10.1037/a0016670

15. Sansone RA, Sansone LA. Workplace bullying: a tale of adverse consequences. Innov Clin Neurosci. 2015;12(1-2):32.

16. Al Omar M, Salam M, Al-Surimi K. Workplace bullying and its impact on the quality of healthcare and patient safety. Hum Resour Health. 2019;17(1):89. doi:10.1186/s12960-019-0433-x

17. Gaffney DA, DeMarco RF, Hofmeyer A, Vessey JA, Budin WC. Making things right: nurses' experiences with workplace bullyinga grounded theory. Nurs Res Pract. 2012;2012:1-10. doi:10.1155/ $2012 / 243210$

18. Srabstein JC, Leventhal BL. Prevention of bullying-related morbidity and mortality: a call for public health policies. SciELO Public Health. 2010;403.

19. Hogh A, Hoel H, Carneiro IG. Bullying and employee turnover among healthcare workers: a three-wave prospective study. $J$ Nurs Manag. 2011;19(6):742-751. doi:10.1111/j.1365-2834.2011.01264.x

20. Almutairi KM. Culture and language differences as a barrier to provision of quality care by the health workforce in Saudi Arabia. Saudi Med J. 2015;36(4):425. doi:10.15537/smj.2015. 4.10133

21. Al-Hanawi MK, Khan SA, Al-Borie HM. Healthcare human resource development in Saudi Arabia: emerging challenges and opportunities -a critical review. Public Health Rev. 2019;40(1):1. doi:10.1186/ s40985-019-0112-4

22. Kvas A, Seljak J. Unreported workplace violence in nursing. Int Nurs Rev. 2014;61(3):344-351. doi:10.1111/inr.12106

23. Leisy HB, Ahmad M. Altering workplace attitudes for resident education (AWARE): discovering solutions for medical resident bullying through literature review. BMC Med Educ. 2016;16(1):127. doi:10.1186/s12909-016-0639-8

24. Tamhane AR, Westfall AO, Burkholder GA, Cutter GR. Prevalence odds ratio versus prevalence ratio: choice comes with consequences. Stat Med. 2016;35(30):5730-5735. doi:10.1002/sim.7059

25. Wang M-L, Hsieh Y-H. Do gender differences matter to workplace bullying? Work. 2016;53(3):631-638. doi:10.3233/WOR-152239

26. Colón-Emeric CS, Ammarell N, Bailey D, et al. Patterns of medical and nursing staff communication in nursing homes: implications and insights from complexity science. Qual Health Res. 2006;16 (2):173-188. doi:10.1177/1049732305284734 
27. Montgomery A, Spânu F, Băban A, Panagopoulou E. Job demands, burnout, and engagement among nurses: a multi-level analysis of ORCAB data investigating the moderating effect of teamwork. Burn Res. 2015;2(2-3):71-79. doi:10.1016/j.burn.2015.06.001

28. Loghmani L, Borhani F, Abbaszadeh A. Factors affecting the nursepatients' family communication in intensive care unit of kerman: a qualitative study. J Caring Sci. 2014;3(1):67.

29. Hawkins N, Jeong S, Smith T. New graduate registered nurses' exposure to negative workplace behaviour in the acute care setting: an integrative review. Int J Nurs Stud. 2019;93:41-54. doi:10.1016/j. ijnurstu.2018.09.020
30. Taylor R. Nurses' perceptions of horizontal violence. Glob Qual Nurs Res. 2016;3:1-9. doi:10.1177/2333393616641002

31. Makarem NN, Tavitian-Elmadjian LR, Brome D, Hamadeh GN, Einarsen S. Assessment of workplace bullying: reliability and validity of an Arabic version of the Negative Acts Questionnaire-Revised (NAQ-R). BMJ Open. 2018;8(12):e024009. doi:10.1136/bmjopen-2018024009

32. Arnetz JE, Fitzpatrick L, Cotten SR, Jodoin C. Workplace bullying among nurses: developing a model for intervention. Violence Vict. 2019;34(2):346-362. doi:10.1891/0886-6708.VV-D-17-00211

\section{Publish your work in this journal}

Risk Management and Healthcare Policy is an international, peerreviewed, open access journal focusing on all aspects of public health, policy, and preventative measures to promote good health and improve morbidity and mortality in the population. The journal welcomes submitted papers covering original research, basic science, clinical \& epidemiological studies, reviews and evaluations, guidelines, expert opinion and commentary, case reports and extended reports. The manuscript management system is completely online and includes a very quick and fair peer-review system, which is all easy to use. Visit http://www.dovepress.com/testimonials.php to read real quotes from published authors. 